\title{
New factorization relations for Yang-Mills amplitudes
}

\author{
N. E. J. Bjerrum-Bohr, ${ }^{1}$ Poul H. Damgaard, ${ }^{1}$ and Humberto Gomez ${ }^{1,2}$ \\ ${ }^{1}$ Niels Bohr International Academy and Discovery Center, Niels Bohr Insitute, \\ University of Copenhagen Blegdamsvej 17, DK-2100 Copenhagen $\varnothing$, Denmark \\ ${ }^{2}$ Facultad de Ciencias Basicas, Universidad Santiago de Cali, Calle 5 N ${ }^{\circ}$ 62-00 Barrio Pampalinda, Cali, Valle, Colombia
}

(Received 17 October 2018; published 28 January 2019)

\begin{abstract}
A double-cover extension of the scattering equation formalism of Cachazo, He, and Yuan leads us to conjecture covariant factorization formulas of $n$-particle scattering amplitudes in Yang-Mills theories. Evidence is given that these factorization relations are related to Berends-Giele recursions through repeated use of partial fraction identities involving linearized propagators.
\end{abstract}

DOI: $10.1103 /$ PhysRevD.99.025014

\section{INTRODUCTION}

The CHY formalism of scattering equations of Cachazo, $\mathrm{He}$, and Yuan provide an intriguing novel way of computing gauge and gravity S-matrix elements [1-3]. The $n$-point scattering amplitudes are expressed here in terms of integrals over auxiliary variables $z_{a}$ on the Riemann sphere that become localized on the set of solutions to the scattering equations,

$$
S_{a} \equiv \sum_{b=1, b \neq a}^{n} \frac{s_{a b}}{z_{a}-z_{b}}=0
$$

Here $s_{a b}=2 k_{a} \cdot k_{b}$ are generalized Mandelstam variables, and the index $a$ labels the (ordered) external particles of momenta $k_{a}$. One remarkable feature of the CHY formalism, and one which shows its fundamental nature, is that it is dimension-agnostic. The defining integral over the variables $z_{a}$ is invariant under an $\operatorname{SL}(2, \mathbf{C})$ transformation

$$
z_{a} \rightarrow \frac{A z_{a}+B}{C z_{a}+D}, \quad A D-B C=1,
$$

which needs to be fixed. Fixing three of the variables in the standard manner, only $(n-3)$ variables $z_{a}$ are left. This precisely matches the $(n-3)$ independent scattering equations after imposing overall momentum conservation. The number of independent solutions $(n-3)$ ! is nevertheless huge, and finding all these solutions is computationally difficult even for moderate values of $n$. Summing over these independent solutions can fortunately be done more directly, through general integration rules developed

Published by the American Physical Society under the terms of the Creative Commons Attribution 4.0 International license. Further distribution of this work must maintain attribution to the author(s) and the published article's title, journal citation, and DOI. Funded by SCOAP ${ }^{3}$. in Refs. [4,5]. A proof of the CHY formalism has been provided by Dolan and Goddard in Ref. [6].

Recently, one of us [7] (see also Ref. [8]) showed how the $\mathrm{CHY}$ formalism can be given a new formulation in which the basic variables $z_{a}$ live not on $\mathbb{C P}^{1}$ but on the complex projective plane $\mathbb{C P}^{2}$. Dubbed the " $\Lambda$-formalism" in [7], here we refer to it as $\mathrm{CHY}$ on a double cover. At first sight it may seem to be a complication to extend the CHY formalism in this manner. However, as we demonstrate in this paper, the double-cover formalism adds a new ingredient to the standard CHY formalism that is much more difficult to extract in the single-cover formulation. Briefly stated, the double-cover formalism naturally expresses the scattering amplitude so that it is factorized into different channels. The propagator that forms the bridge between two factorized pieces arises as the link between two separate $\mathbb{C P}^{1}$ pieces, thus intuitively explaining why the double cover naturally expresses amplitudes in a factorized manner.

In many cases, the factorizations obtained in this way correspond directly to all the physical channels. Interestingly, there are instances where, unavoidably, the factorizations proceed in a slightly different manner: Some physical channels appear immediately, but others only resurface after pole-canceling terms have rearranged the expressions.

We start with a brief review of the CHY formalism and then give the corresponding expressions in the doublecover formulation of Ref. [7]. Next, we describe how the evaluation of amplitudes on a double cover produces factorizations into different channels. Finally, we write down an explicit factorization expression valid for $n$ gluons in any dimension and relate it to known techniques such as on-shell and Berends-Giele recursions.

\section{THE CHY CONSTRUCTION AND A DOUBLE-COVER}

Consider the scattering of $n$ massless particles. The scattering data will then be presented in terms of a set 
of $n$ momentum vectors $\left\{k_{1}^{\mu}, k_{2}^{\mu}, \ldots, k_{n}^{\mu}\right\}$ and $n$ "wave functions" that encode the spin degrees of freedom. For Yang-Mills amplitudes the latter will correspond to the polarization vectors $\left\{\epsilon_{1}^{\mu}, \epsilon_{2}^{\mu}, \ldots, \epsilon_{n}^{\mu}\right\}$. Graviton scattering will similarly be characterized by a set of polarization tensors or, put more simply, as outer products of polarization vectors.

Let us introduce the compact notation of $|i j k|_{z}$ indicating the Vandermonde determinant of variables $z_{i}, z_{j}, z_{k}$ :

$$
|i j k|_{z} \equiv \prod_{i<j}\left(z_{j}-z_{i}\right)=\left|\begin{array}{ccc}
1 & z_{i} & z_{i}^{2} \\
1 & z_{j} & z_{j}^{2} \\
1 & z_{k} & z_{k}^{2}
\end{array}\right| .
$$

It is possible to show that for any rational function $H(z)$ which transforms as

$$
H(z) \rightarrow H(z) \prod_{a=1}^{n}\left(C z_{a}+D\right)^{4},
$$

when

$$
z_{a} \rightarrow \frac{A z_{a}+B}{C z_{a}+D} \quad \text { and } \quad A B-C D=1,
$$

the contour integral [2]

$$
\int \prod_{a=1, a \neq\{i, j, k\}}^{n} d z_{a} \frac{|i j k|_{z}|p q r|_{z}}{\prod_{c=1, c \neq\{p, q, r\}}^{n} S_{c}(z)} H(z)
$$

is independent of the choice of fixed punctures, $\left\{z_{i}, z_{j}, z_{k}\right\}$, and of equations eliminated, $\left\{S_{p}, S_{q}, S_{r}\right\}$.

The precise form of the integrand $H(z)$ defines different (color-ordered) theories. The simplest case is $\phi^{3}$ theory. Let us define a "Parke-Taylor"-factor

$$
P T(1,2, \ldots, n) \equiv \frac{1}{\left(z_{1}-z_{2}\right)\left(z_{2}-z_{3}\right) \cdots\left(z_{n}-z_{1}\right)} .
$$

Color-ordered $\phi^{3}$ amplitudes correspond to integrands with such factors squared:

$$
H(z)=[P T(1,2, \ldots, n)]^{2} .
$$

As shown in Refs. [9,10] (see also [11]), the basic building blocks of other theories are products of one Parke-Taylor factor with a shuffled Parke-Taylor factor ( $\alpha$ indicating a permutation):

$H(z)=P T(1,2, \ldots, n) \times P T(\alpha(1), \alpha(2), \ldots, \alpha(n))$.

Such a product of Parke-Taylor factors in the integrand thus forms a basic skeleton for all other theories.
For Yang-Mills theory we have

$$
H_{n}^{\mathrm{YM}}=P T(1,2, \ldots, n) \times \mathrm{Pf}^{\prime} \Psi_{n},
$$

where

$$
\mathrm{Pf}^{\prime} \Psi_{n} \equiv \frac{(-1)^{i+j}}{z_{i}-z_{j}} \operatorname{Pf}\left[\left(\Psi_{n}\right)_{i j}^{i j}\right] .
$$

The $2 n \times 2 n$ matrix, $\Psi_{n}$, is defined as

$$
\Psi_{n} \equiv\left(\begin{array}{cc}
A & -C^{\mathrm{T}} \\
C & B
\end{array}\right)
$$

with

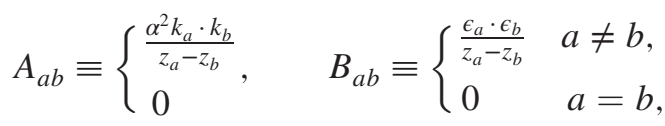

and

$$
C_{a b} \equiv \begin{cases}\frac{\alpha \epsilon_{a} \cdot k_{b}}{z_{a}-z_{b}} & a \neq b, \\ -\sum_{c=1 ; c \neq a}^{n} \frac{\alpha \epsilon_{a} \cdot k_{c}}{z_{a}-z_{c}} & a=b .\end{cases}
$$

Notice the unusual normalization in the $A$ and $C$ matrices. If we put $\alpha=1$, we recover the $\mathrm{CHY}$ prescription as originally defined. If instead we choose $\alpha=\sqrt{2}$, the normalization matches with the color-ordered Feynman rules given by Dixon in [12]. In what follows, $\alpha$ can take any value (it only changes the overall normalization of the color-ordered amplitudes, a convention), but we keep it arbitrary at this point to facilitate a comparison with Feynman diagrams based on color-ordered Feynman rules later in this paper. The matrix $\left(\Psi_{n}\right)_{i j}^{i j}$ denotes the reduced matrix obtained by removing the rows and columns $i, j$ from $\Psi_{n}$, where $1 \leq i<j \leq n$. For how to use the integration rules [5,13] in the context of Yang-Mills theory, see [9-11].

\section{A. The double cover}

A double-cover version of the CHY construction was recently developed by one of us in [7]. In this approach the amplitudes are given as contour integrals on $n$-punctured double-covered Riemann spheres. Restricted to the curves $0=\mathrm{C}_{a} \equiv y_{a}^{2}-\sigma_{a}^{2}+\Lambda^{2}$ for $a=1, \ldots, n$, the pairs $\left(\sigma_{1}, y_{1}\right)$, $\left(\sigma_{2}, y_{2}\right), \ldots,\left(\sigma_{n}, y_{n}\right)$ provide the new set of doubled variables. A translation table has been worked out in detail in Ref. [7]. Specifically, one defines

$$
\tau_{(a, b)} \equiv \frac{1}{2\left(\sigma_{a}-\sigma_{b}\right)}\left(\frac{y_{a}+y_{b}+\sigma_{a}-\sigma_{b}}{y_{a}}\right)
$$

and

$$
\Delta_{(p q r)} \equiv\left(\tau_{(p, q)} \tau_{(q, r)} \tau_{(r, p)}\right)^{-1}
$$


and simultaneously imposes scattering equations in the form (momentum conservation $\sum k_{a}=0$ is implicitly used throughout)

$$
S_{a}^{\tau} \equiv \sum_{\substack{b=1 \\ b \neq a}}^{n} s_{a b} \tau_{(a, b)}=0,
$$

where $a=1, \ldots, n$. Amplitudes are then derived from the following expression:

$$
A_{n}^{\Lambda}=\int_{\Gamma} d \mu_{n}^{\Lambda} \times \frac{\mathcal{I}_{n}(\sigma, y)}{S_{m}^{\tau}},
$$

where the measure $d \mu_{n}^{\Lambda}$ is defined as

$d \mu_{n}^{\Lambda} \equiv \frac{1}{\operatorname{Vol}(\mathrm{GL}(2, \mathbb{C}))} \times \frac{d \Lambda}{\Lambda} \prod_{a=1}^{n} \frac{y_{a} d y_{a} d \sigma_{a}}{\mathrm{C}_{a}} \frac{\Delta_{(p q r)}}{\prod_{d \neq p, q, r, m} S_{d}^{\tau}}$,

with the $\Gamma$ contour being defined by the equations

$$
\left\{\begin{array}{c}
\Lambda=0 \\
S_{d}^{\tau}=0
\end{array} \quad \text { for } d \neq\{p, q, r, m\}, \quad \mathrm{C}_{1}=0, \ldots, \mathrm{C}_{n}=0 .\right.
$$

This rewriting of the amplitude in terms of this contour $\Gamma$, which does not encircle the scattering equation $S_{m}^{\tau}$, follows from the global residue theorem. Note that the integrand now includes a scale $\Lambda$. In order to fix this larger $\operatorname{GL}(2, \mathbb{C})$ symmetry, we gauge-fix four $\sigma_{a}$ 's. Then the measure must be multiplied by the Faddeev-Popov determinant

$$
\Delta_{(p q r \mid m)} \equiv \sigma_{p} \Delta_{(q r m)}-\sigma_{m} \Delta_{(p q r)}+\sigma_{r} \Delta_{(m p q)}-\sigma_{q} \Delta_{(r m p)} .
$$

Therefore, $d \mu_{n}^{\Lambda}$ becomes

$$
d \mu_{n}^{\Lambda}=\frac{1}{2^{2}} \frac{d \Lambda}{\Lambda} \prod_{a=1}^{n} \frac{y_{a} d y_{a}}{\mathrm{C}_{a}} \prod_{d \neq p, q, r, m} \frac{d \sigma_{d}}{S_{d}^{\tau}} \times \Delta_{(p q r \mid m)} \Delta_{(p q r)},
$$

which has been explained in detail in [7,14].

As in the original CHY approach, the precise form of the integrand $\mathcal{I}_{n}(\sigma, y)$ defines the theory. For example, colorordered $\phi^{3}$ theory corresponds to the integrand

$$
\mathcal{I}_{n}=\left[P T^{\tau}(1,2, \ldots n)\right]^{2},
$$

where

$$
P T^{\tau}(1,2, \ldots n) \equiv \tau_{(1,2)} \tau_{(2,3)} \cdots \tau_{(n, 1)} .
$$

Note the $\tau$ 's are neither antisymmetric nor symmetric; the precise definition as given above is correct. Similarly, other theories correspond to products of such modified ParkeTaylor factors with additional expressions, much like in the original CHY formalism. Again, the integrands for these other theories can be broken down to products of shuffled Parke-Taylor expressions.

\section{THE YANG-MILLS THEORY IN THE DOUBLE-COVER PRESCRIPTION}

Since $\tau_{(a, b)} \neq-\tau_{(b, a)}$, it is not immediately obvious how to define the double-cover analog of the reduced Pfaffian for pure Yang-Mills theory. In order to obtain the double-cover version of the $\Psi_{n}$ matrix, we write [we define $\left.(y \sigma)_{a} \equiv y_{a}+\sigma_{a}\right]$

$$
\tau_{(a, b)}=\frac{(y \sigma)_{a}}{y_{a}} \times T_{a b} \equiv \frac{(y \sigma)_{a}}{y_{a}} \times \frac{1}{(y \sigma)_{a}-(y \sigma)_{b}},
$$

on the support, $\mathrm{C}_{a}=\mathrm{C}_{b}=0$, where clearly $T_{a b}=-T_{b a}$. Since $T_{a b}$ is antisymmetric, we establish the single- and double-cover identification, $\frac{1}{\sigma_{a b}} \leftrightarrow T_{a b}$, so the double-cover matrix $\Psi_{n}^{\Lambda}$ is defined as $\left.\Psi_{n}^{\Lambda} \equiv \Psi_{n}\right|_{\frac{1}{\sigma_{a b}} \rightarrow T_{a b}}$. Notice that it is straightforward to rewrite the $\phi^{3}$ integrand in terms of $T_{a b}$, namely,

$$
\mathcal{I}_{n}^{\phi^{3}}(\alpha \mid \beta)=P T^{\tau}\left(\alpha_{1}, \ldots \alpha_{n}\right) \times \prod_{a=1}^{n} \frac{(y \sigma)_{a}}{y_{a}} \times P T^{T}\left(\beta_{1}, \ldots \beta_{n}\right),
$$

with

$$
P T^{T}\left(\beta_{1}, \beta_{2}, \ldots \beta_{n}\right) \equiv T_{\beta_{1} \beta_{2}} T_{\beta_{2} \beta_{3}} \cdots T_{\beta_{n} \beta_{1}} .
$$

Following the CHY program developed in [3], the doublecover representation of the ordered Yang-Mills amplitude is obtained by the replacement $P T^{T}\left(\beta_{1}, \beta_{2}, \ldots \beta_{n}\right) \rightarrow$ $(-1)^{i+j} T_{i j} \operatorname{Pf}\left[\left(\Psi_{n}^{\Lambda}\right)_{i j}^{i j}\right]$, i.e.,

$$
\mathcal{I}_{n}^{\mathrm{YM}}(\alpha)=P T^{\tau}\left(\alpha_{1}, \ldots \alpha_{n}\right) \times \mathrm{Pf}^{\prime} \Psi_{n}^{\Lambda},
$$

with

$$
\operatorname{Pf}^{\prime} \Psi_{n}^{\Lambda} \equiv \prod_{a=1}^{n} \frac{(y \sigma)_{a}}{y_{a}} \times(-1)^{i+j} T_{i j} \operatorname{Pf}\left[\left(\Psi_{n}^{\Lambda}\right)_{i j}^{i j}\right]
$$

where the $\left(\Psi_{n}^{\Lambda}\right)_{i j}^{i j}$ matrix is given by removing the rows and columns $i, j$ from $\Psi_{n}^{\Lambda}$, with $1 \leq i<j \leq n$. Therefore, the pure Yang-Mills amplitude at tree level in the double-cover language is given by the expression

$$
A_{n}(\alpha)=\int_{\Gamma} d \mu_{n}^{\Lambda} \frac{(-1) \Delta_{(p q r)} \Delta_{(p q r \mid m)}}{S_{m}^{\tau}} \times \mathcal{I}_{n}^{\mathrm{YM}}(\alpha)
$$

where the upper index "YM" in $A_{n}(\alpha)$ is no longer necessary. 


\section{A SIMPLE EXAMPLE}

As a simple example, let us consider the four-point amplitude $A_{4}(1,2,3,4)$, with the gauge fixing $(p q r \mid m)=$ $(123 \mid 4)$ and the reduced matrix $\left(\Psi_{4}^{\Lambda}\right)_{13}^{13}$.

First, we focus on the configuration where the sets of punctures $\left(\sigma_{1}, \sigma_{2}\right)$ and $\left(\sigma_{3}, \sigma_{4}\right)$ are on the upper and the lower sheet of the curves, respectively,

$$
\begin{array}{ll}
\left(y_{1}=+\sqrt{\sigma_{1}^{2}-\Lambda^{2}}, \sigma_{1}\right), & \left(y_{2}=+\sqrt{\sigma_{2}^{2}-\Lambda^{2}}, \sigma_{2}\right), \\
\left(y_{3}=-\sqrt{\sigma_{3}^{2}-\Lambda^{2}}, \sigma_{3}\right), & \left(y_{4}=-\sqrt{\sigma_{4}^{2}-\Lambda^{2}}, \sigma_{4}\right) .
\end{array}
$$

Expanding all elements in $A_{4}(1,2,3,4)$ around $\Lambda=0$, we obtain (to leading order)

$$
\begin{aligned}
\left.P T^{\tau}(1,2,3,4)\right|_{3,4} ^{1,2}= & \frac{\Lambda^{2}}{2^{2}} \frac{1}{\left(\sigma_{12} \sigma_{2 P_{34}} \sigma_{P_{34} 1}\right)} \frac{1}{\left(\sigma_{P_{12} 3} \sigma_{34} \sigma_{4 P_{12}}\right)}, \\
\left.\frac{\Delta(123) \Delta(123 \mid 4)}{S_{4}^{\tau}}\right|_{3,4} ^{1,2}= & \frac{2^{5}}{\Lambda^{4}}\left(\sigma_{12} \sigma_{2 P_{34}} \sigma_{P_{34} 1}\right)^{2}\left(\frac{1}{P_{34}^{2}}\right) \\
& \times\left(\sigma_{P_{12} 3} \sigma_{34} \sigma_{4 P_{12}}\right)^{2}
\end{aligned}
$$$$
\left.\prod_{a=1}^{4} \frac{(y \sigma)_{a}}{y_{a}} \times T_{13} \operatorname{Pf}\left[\left(\Psi_{4}^{\Lambda}\right)_{13}^{13}\right]\right]_{3,4}^{1,2}=-\frac{\Lambda^{2}}{2^{2}} \sum_{M} \frac{(-1)}{\sigma_{P_{34}}}
$$$$
\times \operatorname{Pf}\left[\begin{array}{cccc}
0 & -\alpha \frac{\epsilon_{34}^{M} \cdot k_{2}}{\sigma_{342}} & -\alpha \frac{\epsilon_{1} \cdot k_{2}}{\sigma_{12}} & -C_{22} \\
\alpha \frac{\epsilon_{34}^{M} \cdot k_{2}}{\sigma_{P_{34} 2}} & 0 & \frac{\epsilon_{34}^{M} \cdot \epsilon_{1}}{\sigma_{P_{34} 1}} & \frac{\epsilon_{34}^{M} \cdot \epsilon_{2}}{\sigma_{P_{34} 2}} \\
\alpha \frac{\epsilon_{1} \cdot k_{2}}{\sigma_{12}} & \frac{\epsilon_{1} \cdot \epsilon_{34}^{M}}{\sigma_{1 P_{34}}} & 0 & \frac{\epsilon_{1} \cdot \epsilon_{2}}{\sigma_{12}} \\
C_{22} & \frac{\epsilon_{2} \cdot \epsilon_{34}^{M}}{\sigma_{2 P_{34}}} & \frac{\epsilon_{2} \cdot \epsilon_{1}}{\sigma_{21}} & 0
\end{array}\right]
$$$$
\times \frac{(-1)}{\sigma_{P_{12} 3}} \times \operatorname{Pf}\left[\begin{array}{cccc}
0 & -\alpha \frac{\epsilon_{12}^{M} \cdot k_{4}}{\sigma_{P_{12}}} & -\alpha \frac{\epsilon_{3} \cdot k_{4}}{\sigma_{34}} & -C_{44} \\
\alpha \frac{\epsilon_{12}^{M} \cdot k_{4}}{\sigma_{P_{12}}} & 0 & \frac{\epsilon_{12}^{M} \cdot \epsilon_{3}}{\sigma_{P_{12} 3}} & \frac{\epsilon_{12}^{M} \cdot \epsilon_{4}}{\sigma_{P_{12}}} \\
\alpha \frac{\epsilon_{3} \cdot k_{4}}{\sigma_{34}} & \frac{\epsilon_{3} \cdot \epsilon_{12}^{M}}{\sigma_{3 P_{12}}} & 0 & \frac{\epsilon_{3} \cdot \epsilon_{4}}{\sigma_{34}} \\
C_{44} & \frac{\epsilon_{4} \cdot \epsilon_{12}^{M}}{\sigma_{4 P_{12}}} & \frac{\epsilon_{4} \cdot \epsilon_{3}}{\sigma_{43}} & 0
\end{array}\right]
$$$$
=-\frac{\Lambda^{2}}{2^{2}} \sum_{M} \frac{(-1)}{\sigma_{P_{34} 1}} \operatorname{Pf}\left[\left(\Psi_{3}\right)_{P_{34}}^{P_{34} 1}\right] \times \frac{(-1)}{\sigma_{P_{12} 3}} \operatorname{Pf}\left[\left(\Psi_{3}\right)_{P_{12} 3}^{P_{12} 3}\right] \text {, }
$$

where we have introduced the notation $P_{i j} \equiv k_{i}+k_{j}$ and the new fixed punctures $\sigma_{P_{34}}=\sigma_{P_{12}}=0$. The $C_{22}$ and $C_{44}$ factors are given by the usual expressions [15] $C_{22}=-\alpha \frac{\epsilon_{2} \cdot k_{1}}{\sigma_{21}}-\alpha \frac{\epsilon_{2} \cdot P_{34}}{\sigma_{2 P_{34}}}, C_{44}=-\alpha \frac{\epsilon_{4} \cdot k_{3}}{\sigma_{43}}-\alpha \frac{\epsilon_{4} \cdot P_{12}}{\sigma_{4 P_{12}}}$, and the equality in (32) is obtained under the completeness relationship

$$
\sum_{M} \epsilon_{i}^{M \mu} \epsilon_{j}^{M \nu}=\eta^{\mu \nu}
$$

Therefore, the labels sets $\{1,2\}$ and $\{3,4\}$ have been separated.

From the measure $d \mu_{4}^{\Lambda}=\frac{1}{2^{2}} \frac{d \Lambda}{\Lambda}$, we compute the $\Lambda$ integral, and the amplitude becomes

$\left.A_{4}(1,2,3,4)\right|_{3,4} ^{1,2}=\frac{1}{2} \sum_{M} \frac{A_{3}\left(P_{34}^{\epsilon^{M}}, 1,2\right) \times A_{3}\left(P_{12}^{\epsilon^{M}}, 3,4\right)}{P_{12}^{2}}$,

where on the right-hand side the factorized object is given by amplitudes with one leg off shell, as indicated. The overall factor $1 / 2$ cancels out after summing over mirrored configurations, i.e.,

$$
\begin{aligned}
& \left.A_{4}(1,2,3,4)\right|_{3,4} ^{1,2}+\left.A_{4}(1,2,3,4)\right|_{1,2} ^{3,4} \\
& =\sum_{M} \frac{A_{3}\left(P_{34}^{\epsilon^{M}}, 1,2\right) \times A_{3}\left(P_{12}^{\epsilon^{M}}, 3,4\right)}{P_{12}^{2}} .
\end{aligned}
$$

In a similar way, the factorization expansion $\left.A_{4}(1,2,3,4)\right|_{2,3} ^{4,1}$ becomes

$$
\begin{aligned}
& \left.A_{4}(1,2,3,4)\right|_{2,3} ^{4,1}+\left.A_{4}(1,2,3,4)\right|_{4_{, 1}^{2,3}} ^{2,} \\
& \quad=\sum_{M} \frac{A_{3}\left(P_{41}^{\epsilon_{1}^{M}}, 2,3\right) \times A_{3}\left(P_{23}^{\epsilon^{M}}, 4,1\right)}{P_{23}^{2}} .
\end{aligned}
$$

Notice that after starting with the double-cover reduced matrix, $\left(\Psi_{4}^{\Lambda}\right)_{i j}^{i j}=\left(\Psi_{4}^{\Lambda}\right)_{13}^{13}$, the resulting subamplitudes in (35) and (36) have as reduced matrices the ones obtained by removing the rows or columns,

$\{i, j\}=\{$ off-shell puncture $\} \cup(\{$ all punctures $\} \cap\{1,3\})$,

as can be seen in (32).

Finally, besides the two physical factorization expansions around $\Lambda=0$ achieved previously, from the double-cover approach arises a spurious channel given by $\left.A_{4}(1,2,3,4)\right|_{2,4} ^{1,3}$, up to its mirrored configuration. At leading order, this configuration is expanded as

$$
\begin{aligned}
\left.P T^{\tau}(1,2,3,4)\right|_{2,4} ^{1,3}= & \frac{\Lambda^{4}}{2^{4}} \frac{1}{\left(\sigma_{1 P_{24}}^{2} \sigma_{3 P_{24}}^{2}\right)} \frac{1}{\left(\sigma_{2 P_{13}}^{2} \sigma_{4 P_{13}}^{2}\right)}, \\
\left.\frac{\Delta(123) \Delta(123 \mid 4)}{S_{4}^{\tau}}\right|_{2,4} ^{1,3}= & \frac{2^{5}}{\Lambda^{4}}\left(\sigma_{13} \sigma_{3 P_{24}} \sigma_{P_{24}}\right)^{2} \\
& \times\left(\frac{1}{P_{13}^{2}}\right)\left(\sigma_{P_{13} 2} \sigma_{24} \sigma_{4 P_{13}}\right)^{2},
\end{aligned}
$$




$$
\begin{aligned}
& \left.\prod_{a=1}^{4} \frac{(y \sigma)_{a}}{y_{a}} T_{13} \operatorname{Pf}\left[\left(\Psi_{4}^{\Lambda}\right)_{13}^{13}\right]\right]_{2,4}^{1,3} \\
& =-\frac{\alpha^{2} P_{13}^{2}}{2} \frac{\left(\epsilon_{1} \cdot \epsilon_{3}\right)\left(\epsilon_{2} \cdot \epsilon_{4}\right)}{\sigma_{13}^{2} \sigma_{24}^{2}}=\frac{\left(\sigma_{1 P_{24}} \sigma_{3 P_{24}}\right)\left(\sigma_{2 P_{13}} \sigma_{4 P_{13}}\right)}{\sigma_{13} \sigma_{24}} \\
& \times 2\left\{\sum_{L} \frac{(-1)}{\sigma_{P_{24} 1}} \times \operatorname{Pf}\left[\begin{array}{cccc}
0 & -\alpha \frac{\epsilon_{24}^{L} \cdot k_{3}}{\sigma_{P_{24}}} & -\alpha \frac{\epsilon_{1} \cdot k_{3}}{\sigma_{13}} & -C_{33} \\
\alpha \frac{\epsilon_{24}^{L} \cdot k_{3}}{\sigma_{243}} & 0 & \frac{\epsilon_{24}^{L} \cdot \epsilon_{1}}{\sigma_{P_{24} 1}} & \frac{\epsilon_{24}^{L} \cdot \epsilon_{3}}{\sigma_{P_{24}}} \\
\alpha \frac{\epsilon_{1} \cdot k_{3}}{\sigma_{13}} & \frac{\epsilon_{1} \cdot \epsilon_{24}^{L}}{\sigma_{1 P_{24}}} & 0 & \frac{\epsilon_{1} \cdot \epsilon_{3}}{\sigma_{13}} \\
C_{33} & \frac{\epsilon_{3} \cdot \epsilon_{24}^{L}}{\sigma_{3 P_{24}}} & \frac{\epsilon_{3} \cdot \epsilon_{1}}{\sigma_{31}} & 0
\end{array}\right]\right. \\
& \left.\times \frac{(-1)}{\sigma_{P_{13}}} \times \operatorname{Pf}\left[\begin{array}{cccc}
0 & -\alpha \frac{\epsilon_{13}^{L} \cdot k_{4}}{\sigma_{P_{13}}} & -\alpha \frac{\epsilon_{2} \cdot k_{4}}{\sigma_{24}} & -C_{44} \\
\alpha \frac{\epsilon_{13}^{L} \cdot k_{4}}{\sigma_{P_{13}}} & 0 & \frac{\epsilon_{13}^{L} \cdot \epsilon_{2}}{\sigma_{P_{13} 2}} & \frac{\epsilon_{3}^{L} \cdot \epsilon_{4}}{\sigma_{P_{13}}} \\
\alpha \frac{\epsilon_{2} \cdot k_{4}}{\sigma_{24}} & \frac{\epsilon_{2} \cdot \epsilon_{13}^{L}}{\sigma_{2 P_{13}}} & 0 & \frac{\epsilon_{2} \cdot \epsilon_{4}}{\sigma_{24}} \\
C_{44} & \frac{\epsilon_{4} \cdot \epsilon_{13}^{L}}{\sigma_{4 P_{13}}} & \frac{\epsilon_{4} \cdot \epsilon_{2}}{\sigma_{42}} & 0
\end{array}\right]\right\} \\
& =\frac{\left(\sigma_{1 P_{24}} \sigma_{3 P_{24}}\right)\left(\sigma_{2 P_{13}} \sigma_{4 P_{13}}\right)}{\sigma_{13} \sigma_{24}} 2 \times \sum_{L} \frac{(-1)}{\sigma_{P_{24} 1}} \operatorname{Pf}\left[\left(\Psi_{3}\right)_{P_{24}}^{P_{24}}\right] \\
& \times \frac{(-1)}{\sigma_{P_{13} 2}} \operatorname{Pf}\left[\left(\Psi_{3}\right)_{P_{13}}^{P_{13} 2}\right]
\end{aligned}
$$

with $\sigma_{P_{13}}=\sigma_{P_{24}}=0$, and $\sum_{L}$ means a sum over longitudinal degree of freedoms, namely,

$$
\sum_{L} \epsilon_{i}^{L \mu} \epsilon_{j}^{L \nu}=\frac{P_{i}^{\mu} P_{j}^{\nu}}{P_{i} \cdot P_{j}}
$$

Considering the above expansions we are able to integrate the measure $d \mu_{4}^{\Lambda}=\frac{1}{2^{2}} \frac{d \Lambda}{\Lambda}$, so it is straightforward to see that

$$
\begin{aligned}
& \left.A_{4}(1,2,3,4)\right|_{2,4} ^{1,3}+\left.A_{4}(1,2,3,4)\right|_{2,4} ^{1,3} \\
& \quad=-2 \times\left.\sum_{L} \frac{A_{3}\left(P_{34}^{L}, 1,2\right) \times A_{3}\left(P_{12}^{\epsilon^{L}}, 3,4\right)}{P_{12}^{2}}\right|_{2 \leftrightarrow 3} .
\end{aligned}
$$

Therefore, the double-cover approach gives us the four-point factorization relation

$$
\begin{aligned}
A_{4}(1,2,3,4)= & \sum_{M} \frac{A_{3}\left(P_{41}^{\epsilon^{M}}, 2,3\right) \times A_{3}\left(P_{23}^{\epsilon^{M}}, 4,1\right)}{P_{23}^{2}} \\
& +\sum_{M} \frac{A_{3}\left(P_{34}^{\epsilon^{M}}, 1,2\right) \times A_{3}\left(P_{12}^{\epsilon^{M}}, 3,4\right)}{P_{12}^{2}} \\
& -2 \times\left.\sum_{L} \frac{A_{3}\left(P_{34}^{\epsilon^{L}}, 1,2\right) \times A_{3}\left(P_{12}^{\epsilon^{L}}, 3,4\right)}{P_{12}^{2}}\right|_{2 \leftrightarrow 3},
\end{aligned}
$$

where the subamplitudes are given in the single-cover approach with reduced matrices satisfying Eq. (37).

\section{A NEW RELATION FOR YANG-MILLS AMPLITUDES}

We now generalize the new factorization realization obtained from double-cover formalism in the previous section. As will be shown in great detail elsewhere [14], by integrating the double-cover representation of an ordered Yang-Mills amplitude, one is led to the following general formula which factorizes arbitrary $n$-point YangMills amplitudes into a product of (single-cover) $\mathrm{CHY}$ representations of lower-point amplitudes:

$$
\begin{aligned}
A_{n}(1, \ldots, n)= & \sum_{\epsilon_{M}} \frac{A_{3}\left(P_{4: 1}^{\epsilon_{M}}, 2,3\right) \times A_{n-1}\left(P_{2: 3}^{\epsilon_{M}}, 4, \ldots, n, 1\right)}{P_{23}^{2}} \\
& +\sum_{i=4}^{n}\left[\sum_{\epsilon_{M}} \frac{A_{n-i+3}\left(P_{3: i}^{\epsilon_{M}}, i+1, \ldots 1,2\right) A_{i-1}\left(P_{i+1: 2}^{\epsilon_{M}}, 3, \ldots, i\right)}{P_{i+1: 2}^{2}}\right. \\
& \left.-\left.2 \sum_{\epsilon_{L}} \frac{A_{n-i+3}\left(P_{3: i}^{\epsilon_{L}}, i+1, \ldots 1,2\right) A_{i-1}\left(P_{i+1: 2}^{\epsilon_{L}}, 3, \ldots, i\right)}{P_{i+1: 2}^{2}}\right|_{2 \leftrightarrow 3}\right] .
\end{aligned}
$$

To be clear, this factorized form of Yang-Mills amplitudes is a conjecture. What the double-cover formalism produces directly are the first two terms plus contributions that come from linking amplitudes together with scalar degrees of freedom. Miraculously, it appears that these scalar contributions can be exactly represented by gluing two YangMills amplitudes together with longitudinal polarizations only. The technical details of how these manipulations arise will be presented elsewhere [14]. Needless to say, in the factorized form on the right-hand side, the two amplitudes each have one external leg off shell (although still dressed with the corresponding unphysical polarization vector). Gluing these two amplitudes together proceeds through the polarization sums as described in Eqs. (33) and (40). It should also be stressed that the above expression comes from the double-cover formalism with Mobius and scaleinvariance gauge choices $(p q r \mid m)=(123 \mid 4)$ and reduced matrix $\left(\Psi_{n}^{\Lambda}\right)_{13}^{13}$. 
This is important to remark since the above factorization is a gauge-fixing-dependent expression. Of course, the final result, the left-hand side, is the correct full $n$-point amplitude, but the precise factorized form on the right-hand side depends on that generalized gauge fixing. The three punctures which must be fixed in the smaller off-shell Yang-Mills amplitudes are given by the set $\{$ fixed punctures $=$ $(\{$ all punctures $\} \cap\{1,2,3,4\}) \cup\{$ off-shell puncture $\}$, and their reduced matrices are obtained by removing the rows or columns under the rule given in (37). We denote sums of cyclically consecutive external momenta (modulo the total number of external momenta) by $P_{i: j} \equiv k_{i}+k_{i+1}+\cdots+k_{j-1}+k_{j}$. For expressions with only two momenta involved (not necessarily consecutive), we use the shorthand notation $P_{i j} \equiv k_{i}+k_{j}$.

We have denoted the polarization degrees of freedom by $\epsilon_{M}$ and the longitudinal ones by $\epsilon_{L}$. Using the simple identity $\sum_{M} \epsilon_{i}^{M \mu} \epsilon_{j}^{M \nu}=\sum_{T} \epsilon_{i}^{T \mu} \epsilon_{j}^{T \nu}+\sum_{L} \epsilon_{i}^{L \mu} \epsilon_{j}^{L \nu}$, where $\sum_{T} \epsilon_{i}^{T \mu} \epsilon_{j}^{T \nu}=\eta^{\mu \nu}-\frac{P_{i}^{\mu} P_{j}^{\nu}}{P_{i} \cdot P_{j}}$, we can rewrite (43) in terms of transverse $(\mathrm{T})$ and longitudinal $(\mathrm{L})$ polarization vectors,

$$
\begin{aligned}
A_{n}(1, \ldots, n)= & \sum_{\epsilon_{T}} \frac{A_{3}\left(P_{4: 1}^{\epsilon_{T}}, 2,3\right) A_{n-1}\left(P_{2: 3}^{\epsilon_{T}}, 4, \ldots, n, 1\right)}{P_{23}^{2}}+\sum_{i=4, \epsilon_{T}}^{n} \frac{A_{n-i+3}\left(P_{3: i}^{\epsilon_{T}}, i+1, \ldots 1,2\right) A_{i-1}\left(P_{i+1: 2}^{\epsilon_{T}}, 3, \ldots, i\right)}{P_{i+1: 2}^{2}} \\
& -\left.2 \sum_{i=4, \epsilon_{L}}^{n} \frac{A_{n-i+3}\left(P_{3: i}^{\epsilon_{L}}, i+1, \ldots 1,2\right) A_{i-1}\left(P_{i+1: 2}^{\epsilon_{L}}, 3, \ldots, i\right)}{P_{i+1: 2}^{2}}\right|_{2 \leftrightarrow 3}+\sum_{i=4, \epsilon_{L}}^{n} \frac{A_{n-i+3}\left(P_{3: i}^{\epsilon_{L}}, i+1, \ldots 1,2\right) A_{i-1}\left(P_{i+1: 2}^{\epsilon_{L}}, 3, \ldots, i\right)}{P_{i+1: 2}^{2}} \\
& +\sum_{\epsilon_{L}} \frac{A_{3}\left(P_{4: 1}^{\epsilon_{L}}, 2,3\right) A_{n-1}\left(P_{2: 3}^{\epsilon_{L}}, 4, \ldots, n, 1\right)}{P_{23}^{2}}
\end{aligned}
$$

Notice that the poles related to the longitudinal polarization contributions are not physical, and indeed these unphysical poles are canceled by corresponding numerator factors. This is the way local four-point Yang-Mills interactions appear in this formalism.

\section{A. Feynman diagrams and Bern-Carrasco-Johansson (BCJ) numerators}

We first consider how the double-cover representation relates to BCJ numerator identities [16]. From the formula (44), we arrive at

$$
\begin{aligned}
A_{4}(1,2,3,4)= & \sum_{\epsilon_{T}} \frac{A_{3}\left(P_{12}^{\epsilon_{T}}, 3,4\right) \times A_{3}\left(P_{34}^{\epsilon_{T}}, 1,2\right)}{P_{12}^{2}}+\sum_{\epsilon_{T}} \frac{A_{3}\left(P_{23}^{\epsilon_{T}}, 4,1\right) \times A_{3}\left(P_{41}^{\epsilon_{T}}, 2,3\right)}{P_{41}^{2}} \\
& -2 \sum_{\epsilon_{L}}\left[\left.\frac{A_{3}\left(P_{12}^{\epsilon_{L}}, 3,4\right) \times A_{3}\left(P_{34}^{\epsilon_{L}}, 1,2\right)}{P_{12}^{2}}\right|_{2 \leftrightarrow 3}-\frac{A_{3}\left(P_{12}^{\epsilon_{L}}, 3,4\right) \times A_{3}\left(P_{34}^{\epsilon_{L}}, 1,2\right)}{2 P_{12}^{2}}-\frac{A_{3}\left(P_{23} \epsilon_{L}, 4,1\right) \times A_{3}\left(P_{41}^{\epsilon_{L}}, 2,3\right)}{2 P_{41}^{2}}\right] .
\end{aligned}
$$

It is simple to check that in the normalization convention $\alpha=\sqrt{2}$ (corresponding to [12]), the first and second lines are just the conventionally normalized Feynman diagrams ${ }_{1}^{2} Y_{4}^{3}$ and ${ }_{1}^{2} X_{4}^{3}$ and the remainder represents the quartic vertex, namely ${ }_{1}^{2} X_{4}^{3}$. Finally, to obtain the BCJ numerators, we reorganize (45) in the following way:

$$
\begin{aligned}
A_{4}(1,2,3,4)= & \frac{\mathbf{n}_{s}}{P_{12}^{2}}+\frac{\mathbf{n}_{t}}{P_{41}^{2}}, \quad \text { with } \\
\mathbf{n}_{s}= & \sum_{T} A_{3}\left(P_{12}^{\epsilon_{T}}, 3,4\right) \times A_{3}\left(P_{34}^{\epsilon_{T}}, 1,2\right) \\
& +\sum_{L} P_{12}^{2}\left[-\left.\frac{A_{3}\left(P_{12}^{\epsilon_{L}}, 3,4\right) \times A_{3}\left(P_{34}^{\epsilon_{L}}, 1,2\right)}{P_{12}^{2}}\right|_{2 \leftrightarrow 3}\right. \\
& \left.+\frac{A_{3}^{(1)}\left(P_{23}^{\epsilon_{L}}, 4,1\right) \times A_{3}^{(3)}\left(P_{41}^{\epsilon_{L}}, 2,3\right)}{P_{41}^{2}}\right],
\end{aligned}
$$

$$
\begin{aligned}
\mathbf{n}_{t}= & \sum_{T} A_{3}\left(P_{23}^{\epsilon_{T}}, 4,1\right) \times A_{3}\left(P_{41}^{\epsilon_{T}}, 2,3\right) \\
& +\sum_{L} P_{14}^{2}\left[-\left.\frac{A_{3}\left(P_{12}^{\epsilon_{L}}, 3,4\right) \times A_{3}\left(P_{34}^{\epsilon_{L}}, 1,2\right)}{P_{12}^{2}}\right|_{2 \leftrightarrow 3}\right. \\
& \left.+\frac{A_{3}^{(3)}\left(P_{12}^{\epsilon_{L}}, 3,4\right) \times A_{3}^{(1)}\left(P_{34}^{\epsilon_{L}}, 1,2\right)}{P_{12}^{2}}\right]
\end{aligned}
$$

Using the above equation, it is simple to check that we have $\mathbf{n}_{s}-\mathbf{n}_{t}=\mathbf{n}_{u}$, where $\mathbf{n}_{u}$ can be obtained from $\mathbf{n}_{s}$ under the permutation $(1,2,3,4) \rightarrow(1,3,2,4)$. Extending such ideas to a higher number of points should be a possible avenue and would be very interesting.

\section{B. BCFW recursion}

It is interesting to compare the factorizations above with what one would obtain based on Britto-Cachazo-Feng- 
Witten (BCFW) recursion [17]. To illustrate this, consider the five-point amplitude $A_{5}(1,2,3,4,5)$ and introduce the momentum deformation

$k_{2}^{\mu}(z)=k_{2}^{\mu}+z q^{\mu}, \quad k_{3}^{\mu}(z)=k_{3}^{\mu}-z q^{\mu}, \quad z \in \mathbb{C}$,

where $q^{\mu}$ satisfies $k_{2} \cdot q=k_{3} \cdot q=q \cdot q=0$ and $q \cdot \bar{q}=1$. Additionally, the polarization vectors $\left\{\epsilon_{2}, \epsilon_{3}\right\}$ must be deformed in order to keep the transversality, so we consider $\epsilon_{2}^{+}(z)=\bar{q}-z \frac{k_{3}}{k_{2} \cdot k_{3}}$ and $\epsilon_{3}^{+}(z)=q$; another option is $\epsilon_{2}^{-}(z)=q$ and $\epsilon_{3}^{-}(z)=\bar{q}+z \frac{k_{2}}{k_{2} \cdot k_{3}}$. Since we have momentum conservation for deformed momenta $k_{1}+k_{2}(z)+k_{3}(z)+k_{4}+k_{5}=0$ and the on-shell condition $k_{2}^{2}(z)=k_{3}^{2}(z)=0$ and transversality remain valid, the CHY approach is well defined. Thus, from (43) and using Cauchy, one has

$$
\begin{aligned}
& A_{5}(1,2,3,4,5) \\
& =-\operatorname{Res}_{P_{34}^{2}(z)=0}\left[\frac{\sum_{M} A_{3}\left(P_{5: 2}^{\epsilon_{M}}, 3,4\right)(z) \times A_{4}\left(P_{34}^{\epsilon_{M}}, 5,1,2\right)(z)}{z P_{34}^{2}(z)}\right] \\
& -\operatorname{Res}_{P_{3: 5}^{2}(z)=0}\left[\frac{\sum_{M} A_{3}\left(P_{3: 5}^{\epsilon_{M}}, 1,2\right)(z) A_{4}\left(P_{12}^{\epsilon_{M}}, 3,4,5\right)(z)}{z P_{3: 5}(z)}\right] \\
& -\operatorname{Res}_{z=\infty}\left[\frac{A_{5}(1,2,3,4,5)(z)}{z}\right] .
\end{aligned}
$$

Obviously, the pole $P_{23}^{2}$ does not depend on $z$, so this physical factorization channel only contributes at infinity. The most interesting observation is that the spurious poles $\left.P_{i+1: 2}^{2}(z)\right|_{2 \leftrightarrow 3}$ cancel out because the longitudinal contributions $\sum_{i=4, \epsilon_{L}}^{n} A_{n-i+3}\left(P_{3: i}^{\epsilon_{L}}, i+1, \ldots, 1,2\right) \times A_{i-1}\left(P_{i+1: 2}^{\epsilon_{L}}\right.$, $3,4, \ldots, i)\left.\right|_{2 \leftrightarrow 3}$ are proportional to them. Therefore, the boundary contributions at $z=\infty$ are related to the unphysical poles that appear in the double cover, Eq. (43). This gives these poles a special significance in the context of BCFW recursion and potentially a new recursive path for dealing with such contributions.

\section{Berends-Giele recursion and the double cover}

Another natural question that arises concerns the similarity of the factorized forms from the double-cover method and Berends-Giele recursion [18]. In order to shed light on this, we focus on the bi-adjoint $\phi^{3}$ theory in the double-cover formalism. Because of the trivial numerator factors of this case, it is far simpler to analyze.

The connection is well illustrated by considering the five-point amplitude. The factorizations from the doublecover method lead to

$$
\begin{aligned}
A^{\phi^{3}}(1,2,3,4,5)= & \frac{A_{4}^{\phi^{3}}\left(P_{12}, 3,4,5\right)}{P_{3: 5}^{2}}+\frac{A_{4}^{\phi^{3}}\left(1, P_{23}, 4,5\right)}{P_{4: 1}^{2}} \\
& +\frac{A_{4}^{\phi^{3}}\left(1,2, P_{34}, 5\right)}{P_{34}^{2}} \\
= & \frac{1}{P_{3: 5}^{2}}\left(\frac{1}{P_{5: 2}^{2}-P_{12}^{2}}+\frac{1}{P_{45}^{2}}\right) \\
& +\frac{1}{P_{34}^{2}}\left(\frac{1}{P_{51}^{2}}+\frac{1}{P_{3: 5}^{2}-P_{34}^{2}}\right) \\
& +\frac{1}{P_{4: 1}^{2}}\left(\frac{1}{P_{51}^{2}}+\frac{1}{P_{45}^{2}}\right),
\end{aligned}
$$

where we have chosen the gauge fixing $(p q r \mid m)=$ $(123 \mid 4)$. On the other hand, Berends-Giele recursion gives (see, e.g., Ref. [19])

$\frac{1}{P_{2: 4}^{2}}\left(\frac{1}{P_{34}^{2}}+\frac{1}{P_{23}^{2}}\right)+\frac{1}{P_{12}^{2} P_{34}^{2}}+\frac{1}{P_{1: 3}^{2}}\left(\frac{1}{P_{12}^{2}}+\frac{1}{P_{23}^{2}}\right)$.

On the support, $k_{1}+k_{2}+k_{3}+k_{4}+k_{5}=0$, and under the on-shell condition $k_{i}^{2}=0$, it is trivial to check that the expressions obtained in (49) and (50) are identical. However, the appearance of the unphysical poles in the double-cover framework, $\left(P_{5: 2}^{2}-P_{12}^{2}\right)^{-1}=\left(P_{34}^{2}-P_{3: 5}^{2}\right)^{-1}$ and $\left(P_{3: 5}^{2}-P_{34}^{2}\right)^{-1}$, makes it clear that the two representations are not directly equal. Interestingly, these unphysical poles are related to the physical channel $\frac{1}{P_{3: 5}^{2} P_{34}^{2}}$ by use of the partial fraction identity

$$
\frac{1}{P_{3: 5}^{2} P_{34}^{2}}=\frac{1}{P_{3: 5}^{2}\left(P_{34}^{2}-P_{3: 5}^{2}\right)}+\frac{1}{P_{34}^{2}\left(P_{3: 5}^{2}-P_{34}^{2}\right)} .
$$

As it happens with the linear propagators at loop level [8,20-22], the CHY formalism is naturally built of linear propagators that can relate to the usual Feynman propagators by means of partial fractioning.

\section{CONCLUSIONS}

We have presented a new set of factorization identities for Yang-Mills theory that naturally arise from a doublecover version of the $\mathrm{CHY}$ formalism. These factorizations glue amplitudes together in what can be interpreted as the covariant Feynman gauge, with the additional four-point contact interactions coming from an explicit sum over longitudinal polarizations. The factorizations are at the conjectured level, but there are many hints that they may also be derivable from Berends-Giele recursions. Although spurious poles appear, simple checks show that they cancel through repeated use of partial fraction identities. It would be an interesting extension of this work to derive these relations directly from off-shell recursion relations. 
Factorizations of amplitudes grow out of the doublecover formalism precisely because it is "double": There are, figuratively speaking, two CHY integrals involved. The bridge between these two CHY integrals is an off-shell leg, a propagator. In the double-cover formalism this off-shell leg stems from one scattering equation that is not imposed as a delta-function constraint.

These factorizations of Yang-Mills amplitudes are just a small part of more general relations that follow when the double-cover formalism of CHY is analyzed for the known set of theories that can be represented in this form. Details will be provided by one of us in a subsequent paper [14].

\section{ACKNOWLEDGMENTS}

Numerous discussions with J. Bourjaily are gratefully acknowledged. We also thank N. Ahmadiniaz, C. Cardona, and C. Lopez-Arcos for useful discussions and comments. This work was supported in part by the Danish National Research Foundation (DNRF91). H. G. is grateful for partial financial support from Universidad Santiago de Cali.
[1] F. Cachazo, S. He, and E. Y. Yuan, Scattering equations and Kawai-Lewellen-Tye orthogonality, Phys. Rev. D 90, 065001 (2014).

[2] F. Cachazo, S. He, and E. Y. Yuan, Scattering of Massless Particles in Arbitrary Dimensions, Phys. Rev. Lett. 113, 171601 (2014).

[3] F. Cachazo, S. He, and E. Y. Yuan, Scattering of massless particles: Scalars, gluons and gravitons, J. High Energy Phys. 07 (2014) 033.

[4] F. Cachazo and H. Gomez, Computation of contour integrals on $\mathcal{M}_{0, n}$, J. High Energy Phys. 04 (2016) 108.

[5] C. Baadsgaard, N. E. J. Bjerrum-Bohr, J. L. Bourjaily, and P. H. Damgaard, Integration rules for scattering equations, J. High Energy Phys. 09 (2015) 129.

[6] L. Dolan and P. Goddard, Proof of the formula of Cachazo, He and Yuan for Yang-Mills tree amplitudes in arbitrary dimension, J. High Energy Phys. 05 (2014) 010.

[7] H. Gomez, $\Lambda$ scattering equations, J. High Energy Phys. 06 (2016) 101.

[8] C. Cardona and H. Gomez, Elliptic scattering equations, J. High Energy Phys. 06 (2016) 094.

[9] N. E. J. Bjerrum-Bohr, J. L. Bourjaily, P. H. Damgaard, and B. Feng, Analytic representations of Yang-Mills amplitudes, Nucl. Phys. B913, 964 (2016).

[10] N. E. J. Bjerrum-Bohr, J. L. Bourjaily, P. H. Damgaard, and B. Feng, Manifesting color-kinematics duality in the scattering equation formalism, J. High Energy Phys. 09 (2016) 094.

[11] C. Cardona, B. Feng, H. Gomez, and R. Huang, Cross-ratio identities and higher-order poles of CHY-integrand, J. High Energy Phys. 09 (2016) 133.

[12] L. J. Dixon, Calculating scattering amplitudes efficiently, arXiv:hep-ph/9601359.

[13] C. Baadsgaard, N. E. J. Bjerrum-Bohr, J. L. Bourjaily, and P. H. Damgaard, Scattering equations and Feynman diagrams, J. High Energy Phys. 09 (2015) 136.
[14] H. Gomez, Scattering equations and a new factorization for amplitudes I: Gauge theories, arXiv:1810.05407.

[15] It is useful to recall that $\epsilon_{2} \cdot P_{34}=-\epsilon_{2} \cdot k_{1}$ and $\epsilon_{4} \cdot P_{12}=$ $-\epsilon_{4} \cdot k_{3}$.

[16] Z. Bern, J. J. M. Carrasco, and H. Johansson, New relations for gauge-theory amplitudes, Phys. Rev. D 78, 085011 (2008).

[17] R. Britto, F. Cachazo, B. Feng, and E. Witten, Direct Proof of Tree-Level Recursion Relation in Yang-Mills Theory, Phys. Rev. Lett. 94, 181602 (2005).

[18] F. A. Berends and W. T. Giele, Recursive calculations for processes with $\mathrm{n}$ gluons, Nucl. Phys. B306, 759 (1988).

[19] C. R. Mafra, Berends-Giele recursion for doublecolor-ordered amplitudes, J. High Energy Phys. 07 (2016) 080.

[20] Y. Geyer, L. Mason, R. Monteiro, and P. Tourkine, Loop Integrands for Scattering Amplitudes from the Riemann Sphere, Phys. Rev. Lett. 115, 121603 (2015); One-loop amplitudes on the Riemann sphere, J. High Energy Phys. 03 (2016) 114; Two-loop scattering amplitudes from the Riemann sphere Phys. Rev. D 94, 125029 (2016); C. Cardona and H. Gomez, CHY-graphs on a torus, J. High Energy Phys. 10 (2016) 116; H. Gomez, S. Mizera, and G. Zhang, CHY loop integrands from holomorphic forms, J. High Energy Phys. 03 (2017) 092.

[21] F. Cachazo, S. He, and E. Y. Yuan, One-loop corrections from higher dimensional tree amplitudes, J. High Energy Phys. 08 (2016) 008.

[22] C. Baadsgaard, N. E. J. Bjerrum-Bohr, J. L. Bourjaily, P. H. Damgaard, and B. Feng, Integration rules for loop scattering equations, J. High Energy Phys. 11 (2015) 080; C. Baadsgaard, N. E. J. Bjerrum-Bohr, J. L. Bourjaily, S. Caron-Huot, P. H. Damgaard, and B. Feng, New Representations of the Perturbative S-Matrix, Phys. Rev. Lett. 116, 061601 (2016). 